\title{
Simulation of Diffusion of a Single Schrödinger Particle in the Quantum Register
}

\author{
M. Ostrowski \\ Institute of Information Technology FTIMS, Technical University of Łódź, \\ Wólczańska 215, 90-924 Łódź, Poland \\ (Received February 6, 2020; in final form March 12, 2020)
}

\begin{abstract}
This paper examines whether a quantum computer can efficiently simulate the evolution of the Schrödinger particle in a one-dimensional infinite potential well. The present study involves simulation of the change of the particle's state due to instant enlarging of the well's size. In order to solve the Schrödinger equation in the quantum register, an algorithm based on the Quantum Fourier Transform is applied. The paper presents an algorithm for simulating time evolution of such a system, implemented on standard two-input gates.
\end{abstract}

DOI: 10.12693/APhysPolA.137.1182

PACS/topics: quantum computations, quantum simulations, Schrödinger particle

\section{Introduction}

In the near future, quantum calculations can make a major contribution to the development of informatics [1]. Although practical implementations of quantum computer have not been built yet, its existence seems to be possible. Therefore, it is worth examining the properties of such machines.

Today we know Shor [2] and Grover [3] algorithms which are of lower computational complexity than their best classical counterparts. Another promising application of quantum computer are quantum simulations [4-6], i.e., the computer modeling of behavior of physical quantum systems. It gives the possibility of effective modeling quantum processes, which is not possible using classical computers [7]. Quantum computers can simulate a wide variety of quantum systems, including fermionic lattice models $[8,9]$, quantum chemistry $[10,11]$, and quantum field theories [12].

This work is a continuation of the autor's previous reserch. Papers $[13,14]$ concerned the simulation of the Schrödinger particle scattered on a rectangular potential. The above-mentioned works focused on the simulation of the particle with a continuous spectrum of energy (in an unlimited space). The present study seeks to determine whether the proposed algorithm, after some modifications, is able to simulate the Schrödinger particle in a closed area, where the spectrum of energy is discrete. We examine a particle trapped in an infinity potential well with a length $a$. As the initial state of the particle one of the stationary states is chosen. Next, we investigate the process of wave function expansion caused by instant enlarging of the size of the well from the initial length $a$ to the final length equal to $2 a$ (Fig. 1).

e-mail: marcin.ostrowski@p.lodz.pl

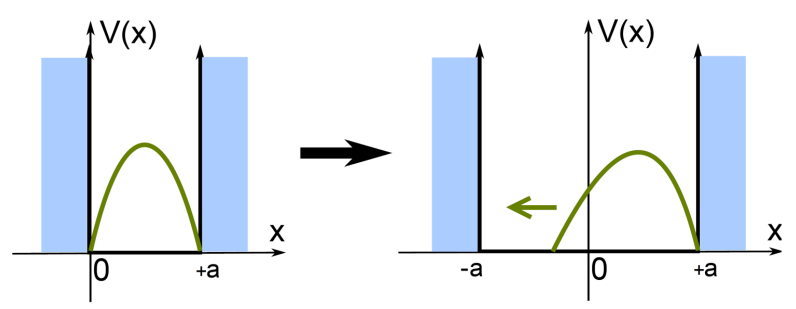

Fig. 1. Diffusion of the Schrödinger particle after an abrupt increase of length of the infinity potential well.

In our study methods based on the quantum Fourier transform are used. Their key advantages are: time efficiency and effective use of the quantum register capacity. In one base state of the register one sample of the wave function is stored, which gives the possibility to store $2^{n_{q}}$ samples in the $n_{q}$ qubit register. However, this approach has also disadvantages. The main limitation of this coding method of the particle state is that it does not enable implementing an arbitrary potential $V(x)$. It allows only a few special cases such as the $V(x) \sim x^{2}$ potential. This case was tested in [15-18], which examined the free particle and the harmonic oscillator. In our previous works we have shown that also rectangular potentials (like thresholds and wells) can be simulated using this method. This provides the ability to examine other interesting processes, such as the tunnel effect [13] or scattering of two particles on each other [14]. The present study also makes use of this kind of potential to simulate a particle with a discrete energy spectrum.

The main motivation of this work is checking if such processes can be effectively simulated in a real quantum register consisting a small (around 10-16) number of qubits. A problem with a known analytical solution (see Appendix) has been intentionally chosen for the present analysis - to enable validation of results. This provides certainty that the results obtained in a real quantum register will be correct. 
A 10-qubit register is sufficient for executing the presented algorithm. However, the algorithm is scalable an increase in the number of qubits means a higher sampling density of the wave function and, consequently, more accurate results. Moreover, standard quantum gates, like NOT-gate and controlled phase-shift gates with precision close to float number (mantissa $2^{-23}$ which gives accuracy of phase argument $\Delta \phi$ at the level of the order of $10^{-7}$ ) are needed. We expect that such quantum computers will be built in the near future, perhaps with the help of semiconductor [19] or quantum dots $[20,21]$ technology. In addition, it seems interesting to build a topological quantum computer $[22,23]$ with anyons [24]. However, this paper describes an algorithm that is completely independent of the physical implementation of the hardware.

The possibility of using a quantum register to simulate the Schrödinger particle has already been discussed in the literature. For example, in $[25,26]$ different technique based on the quantum lattice-gas model was examined. The possibility of simulation diffusion processes in the quantum computer has also been examined. It is worth mention here paper [27], where a lattice-gas algorithm for the one-dimensional diffusion equation is realized using radio frequency pulses in a one-dimensional spin system. Our approach is completely different. We examine the problem on purely algorithmic grounds, using abstract model of quantum system. We abstract completely from specified physical implementation of the quantum register.

\section{The algorithm simulating time evolution of the system}

Time evolution of the Schrödinger particle in a onedimensional case takes the form

$$
\mathrm{i} \hbar \frac{\mathrm{d}}{\mathrm{d} t} \Psi(x, t)=\left(H_{0}+V(x)\right) \Psi(x, t),
$$

where $H_{0}=p^{2} /\left(2 m_{c}\right)$ is free Hamiltonian and $V(x)$ is the potential describing the interactions with an external force. In the present considerations, it is the potential of the well from Fig. 1.

In the present method, the wave function of the particle $\Psi(x)$ is sampled and encoded in the state of the quantum register in the following way:

$$
\psi_{k}=\Psi(k \Delta x-a),
$$

where $\psi_{k}$ is probability amplitude of $k$-th base state of the register $\left(k=0 \ldots, 2^{n_{q}}-1\right), \Delta x=4 a / 2^{n_{q}}$ is sampling interval and $n_{q}$ is number of qubits in the register. Thus, sample $\psi_{0}$ corresponds to $\Psi(-a)$, while sample $\psi_{2^{n_{q}-1}}$ corresponds to $\Psi(3 a-\Delta x)$. In the $x \in(a, 3 a)$ area walls of the well are simulated $(V(x) \rightarrow \infty)$. A single interval is sufficient because the entire simulated area has a circle topology $(\psi(-a)=\psi(3 a))$.

The time evolution operator corresponding to (1) is approximated as follows:

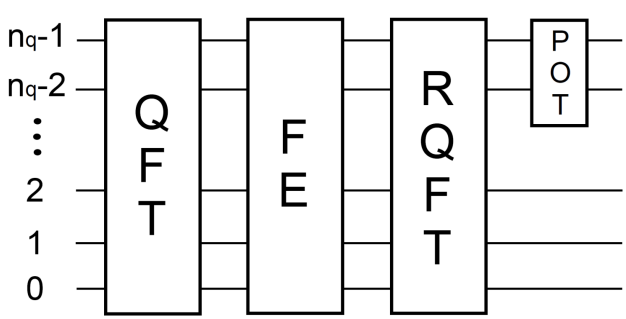

Fig. 2. Scheme of the algorithm simulating the Schrödinger particle in an infinite potential well.

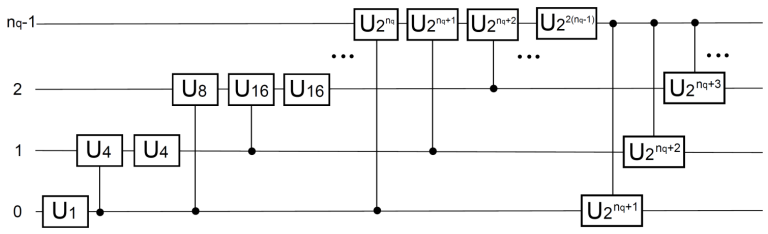

Fig. 3. Scheme of the FE block. Gates labeled by $U_{k}$ are (controlled) phase shift gates with angle $\phi_{k}=$ $-\pi^{2} k \hbar \Delta t /\left(8 m_{c} a^{2}\right)$.
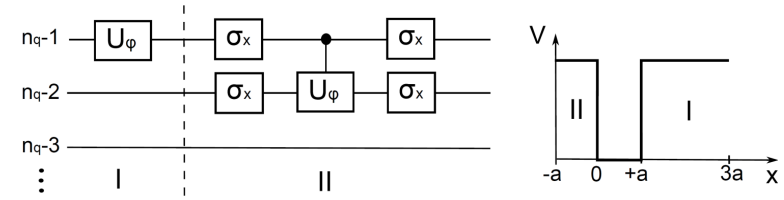

Fig. 4. Scheme of the POT block. The well of length $2 a$ is implemented by the part of algorithm denoted by a Roman numeral I. The well of length $a$ is implemented by parts denoted by I and II.

$$
\begin{gathered}
U(\Delta t) \psi_{k}(t)=\exp \left(-\mathrm{i}\left(H_{0}+V(x)\right) \Delta t / \hbar\right) \psi_{k}(t)= \\
\mathrm{e}^{-\mathrm{i} V(x) \Delta t / \hbar} \mathcal{F}^{-1}\left\{\mathrm{e}^{-\frac{\mathrm{i} p^{2}}{2 m_{c} \hbar}} \mathcal{F}\left\{\psi_{k}(t)\right\}\right\},
\end{gathered}
$$

where $\mathcal{F}$ is the quantum Fourier transform.

The process of simulation is shown in Fig. 2. Blocks QFT, FE, RQFT and POT implement one time step of the simulation (evolution operator from (3). Block QFT and RQFT implement the quantum Fourier transform and its inverse. The implementation of QFT and RQFT blocks on a two-qubit logical gates is presented in [13].

Block FE implements operator $\exp \left(-\mathrm{i} p^{2} / 2 m_{c} \hbar\right)$. Its implementations are shown in Fig. 3. It has been successfully used and described in our earlier works (see [13]). Block POT implements operator $\exp (-\mathrm{i} V(x) \Delta t / \hbar)$. Its implementation is shown in Fig. 4. Gates $U_{\phi}$ are phase shift gates $\left(|1\rangle \rightarrow \mathrm{e}^{\mathrm{i} \phi}|1\rangle\right)$, and controlled phase shift gates $\left(|1,1\rangle \rightarrow \mathrm{e}^{\mathrm{i} \phi}|1,1\rangle\right)$. Gates denoted by $\sigma_{x}$ are the standard NOT gates. In the case of a big well (denoted by the Roman numeral I in Fig. 4) the operation of POT block consists in the multiplication of state $|1\rangle$ of the oldest qubit $\left(n_{q}-1\right)$ by phase-shift factor $\exp (\phi)$, 
where $\phi=V \Delta t / \hbar, V$ is a sufficiently high potential outside the well, and $\Delta t$ is the time step of the simulation. The manner of operation of POT block results from the state coding method given by (2). The state of $n_{q}-1$ qubit determines if the particle stays in $x \in(-a,+a)$ region (for $|0\rangle)$ or $x \in(+a, 3 a)$ region (for $|1\rangle$ ).

\section{The simulation results}

The algorithm is examined for $n_{q}=10$ qubits. As an initial state of simulated particle we choose a stationary states inside the small well given by $(6)$. The results are shown in Figs. 5-9.

Figures 5-8 show the probability distribution for the particle inside the well. On the $x$ axis, the numbers of the space samples are denoted. Sample number 0 corresponds to $x=-a$, sample number 256 to $x=0$ and sample number 512 to $x=+a$. The values on the $y$ axis represent density of the spatial probability

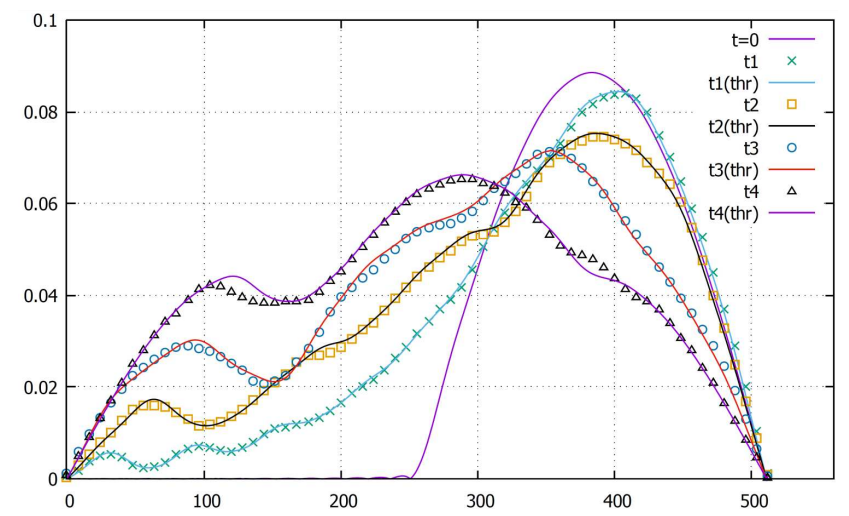

Fig. 5. Evolution of the ground state $(n=1$ from Eq. (6)). Plot shows the spatial probability distribution for the particle in the well for $t=0$ (the moment of enlarging the well), $t_{1}=\frac{1}{4} \frac{T}{16}, t_{2}=\frac{1}{2} \frac{T}{16}, t_{3}=\frac{3}{4} \frac{T}{16}$, and $t_{4}=\frac{T}{16}$.

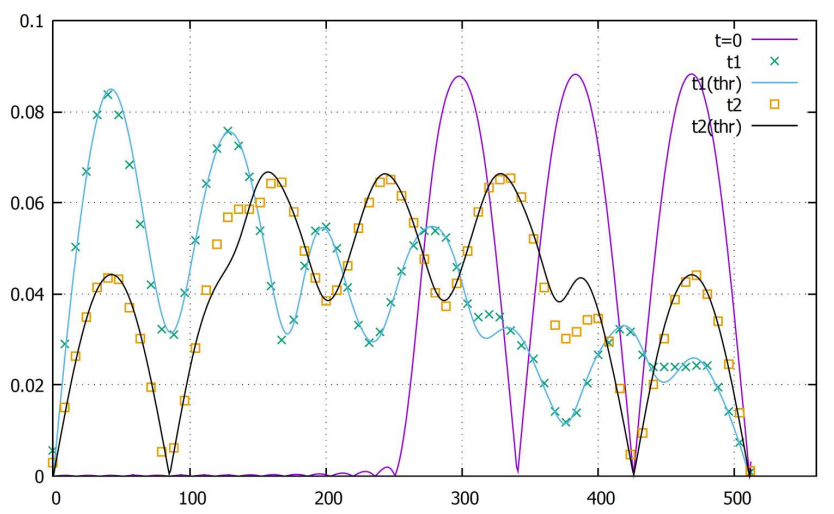

Fig. 6. Evolution of the second excited state $(n=3)$. Plot shows the spatial probability distribution for the particle in the well for $t=0, t_{1}=\frac{1}{2} \frac{T}{16}$ and $t_{2}=\frac{T}{16}$.

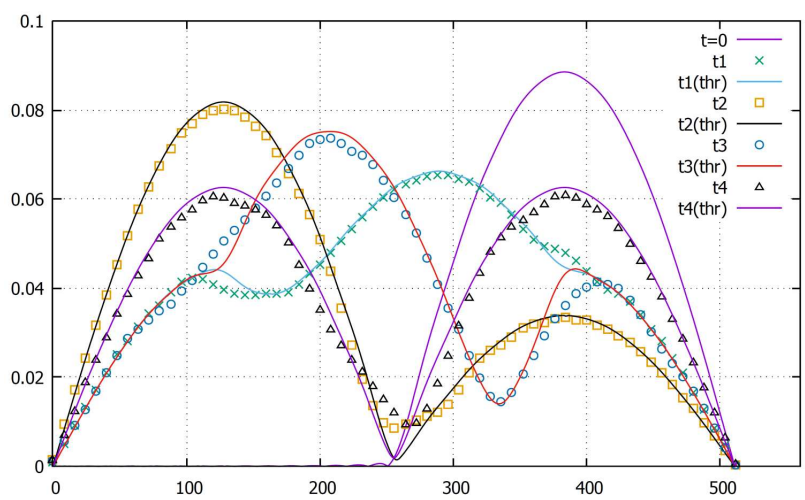

Fig. 7. Evolution of the ground state $(n=1$ from Eq. (6)). Plot shows spatial probability distribution for the particle in the well for $t=0$ (the moment of enlarging the well), $t_{1}=\frac{1}{4} \frac{T}{4}, t_{2}=\frac{1}{2} \frac{T}{4}, t_{3}=\frac{3}{4} \frac{T}{4}$, and $t_{4}=\frac{T}{4}$.

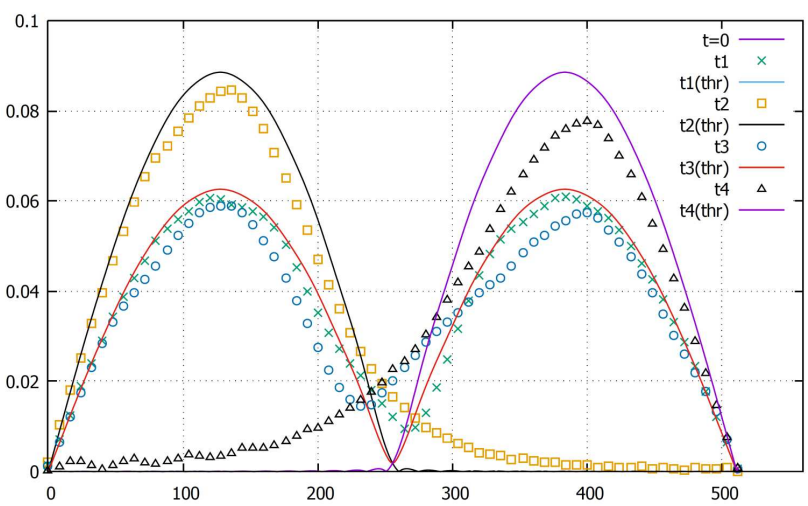

Fig. 8. Evolution of the ground state $(n=1$ from Eq. (6)). The plot shows spatial probability distribution for the particle in the well for $t=0, t_{1}=\frac{1}{4} T$, $t_{2}=\frac{1}{2} T, t_{3}=\frac{3}{4} T$, and $t_{4}=T$.

distribution. All figures are made for $a=10^{-6} \mathrm{~m}$ and $m_{c}=1.67 \times 10^{-27} \mathrm{~kg}$ which corresponds to period $T=8.08 \times 10^{-5} \mathrm{~s}$. Time step of the simulation is equal to $\mathrm{d} t=T /\left(3.2 \times 10^{5}\right)$. Figures $5-8$ demonstrate both the simulation results (doted lines) and the theoretical predictions (solid lines) obtained from (10) in Appendix. Additionally, in each figure legend, theoretical solid lines are marked with the symbol "thr".

As expected, periodic evolution of the system is obtained, with period $T$ given by Eq. (11). This is also visible in Fig. 9. Note that for time $t=\frac{T}{2}(t=0.75 \mathrm{~T}$ in Fig. 9) the wave function of the particle lies completely in the left half of the well, and for time $t=T(t=1.25 \mathrm{~T}$ from Fig. 9) the particle returns again to the right half of the well.

Figure 10 shows the dependence between the register size $\left(n_{q}\right)$ and the accuracy of the obtained results. The tests were carried out for $n_{q}$ from 6 to 12 qubits. The well and the particle parameters are the same as in the previous cases. 


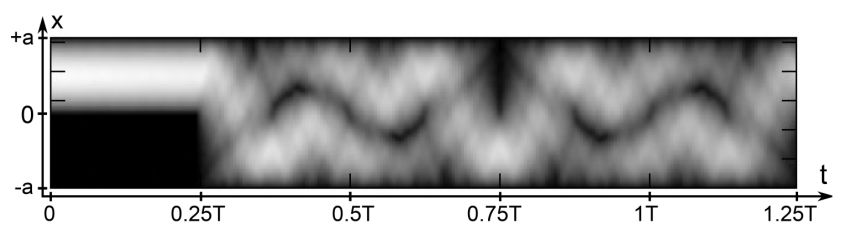

Fig. 9. Evolution of the ground state $(n=1$ from Eq. (6)). The plot shows the spatial probability distribution of the particle as a function of time. The horizontal axis represents time of the simulation, whereas the vertical axis represents $x$-coordinate inside the well. For a point in time $t=0.25 T$ the potential well becomes enlarge. The total time of the simulation is equal to $t=1.25 T=1.01 \times 10^{-4}[\mathrm{~s}](500000$ time samples $)$.

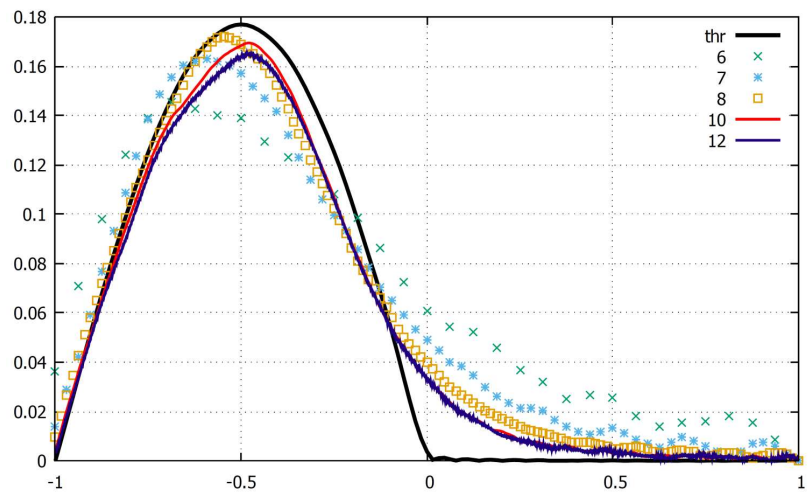

Fig. 10. Spatial probability distribution for the particle in the well for $t=T / 2$. Curve denoted by "thr" is theoretical prediction (from Eq. (10)). Other curves (denoted by numbers) are simulation results. The values on the labels correspond to the size of the register $\left(n_{q}\right)$ used in the simulation. The horizontal axis represents $x$-coordinate (measured in $\mu \mathrm{m}$ ), whereas the vertical axis represents spatial probability distribution (in square root scale for $n_{q}=8$ ).

\section{Conclusions}

- For small periods of time (which are appropriately smaller than the period $T$ ) and for low values of $n$ (the number of stationary states in the well before enlarging) the algorithm is capable of obtaining satisfactory results even for small registers $\left(n_{q}=10\right.$ qubits). In this case, the error obtained from calculating probability of finding a particle on the left or on the right part of the well is smaller than $5 \%$.

- For time $t$ which is comparable to period $T$, and for larger values of $n$, the error can reach up to several dozen percent. In this case greater registers and smaller time steps $\mathrm{d} t$ should be used.

- As can be observed in Fig. 10, increase the number of qubits from 6 to 10 improves the quality of the obtained results. In the case of $n_{q}=12$ qubits, no further improvement is observed. This is due to numerical errors appearing as a noise on the plot.
In order to further improvement of the algorithm accuracy the precision of the numerical representation of the wave function and value of time step $\mathrm{d} t$ should be improved.

- The algorithm is examined for $n_{q}=10$ qubits. It corresponds to a lattice of $2^{9}=512$ the wave function spatial samples. (One qubit is used to the well walls simulation.) As our previous works show (e.g., [13]), classic simulation (Cayley's method) performed on the same lattice with the same time step $\mathrm{d} t$ gives results with similar precision. In this work, we do not present results of the classic comparative simulation because an analytical solution of the problem (10) is available.

- Preparation of the initial state was excluded from the discussion. A simple algorithm for inputting these types of states into the quantum register has been proposed in [28]. Such problem have also been considered in other works, including both standard approaches [29] and those based on genetic algorithms [30].

- Problem of reading of the final data from the register was also excluded from the discussion. We have focused only on whether the final state is correct. In practice, by measuring state of $n_{q}-2$ qubit we are able to determine if a particle is located in $x \in(-a, 0)$ area (if we obtain state $|0\rangle)$ or in $x \in(0,+a)$ area (for state $|1\rangle$ ). Performing the measurement on all qubits, we obtain information about the position of the particle inside the well with an accuracy equal to $\Delta x$. This results from the state coding method given by (2).

- We have also omitted the factors affecting the accuracy of the results such as the accuracy of quantum gates realization or noise in the register (decoherence).

\section{Appendix}

The eigenstates of the Hamiltonian of the particle inside the big well $x \in\langle-a, a\rangle$ in position representation take the form

$$
\left\langle x \mid E_{m}\right\rangle= \begin{cases}\frac{1}{\sqrt{a}} \cos \left(\frac{\pi m x}{2 a}\right) & \text { for } m=1,3,5 \ldots \\ \frac{1}{\sqrt{a}} \sin \left(\frac{\pi m x}{2 a}\right) & \text { for } m=2,4,6 \ldots\end{cases}
$$

The eigenvalues of the Hamiltonian of the particle inside the big well are equal to

$$
E_{m}=\frac{\pi^{2} \hbar^{2}}{8 m_{c} a^{2}} m^{2}
$$

where $m_{c}$ is the mass of the particle.

The eigenstates of the Hamiltonian of the particle inside the small well $x \in\langle 0, a\rangle$ in position representation take the form:

$$
\left\langle x \mid \tilde{E}_{n}\right\rangle=\sqrt{\frac{2}{a}} \sin \left(\frac{\pi n x}{a}\right) \text { for } n=1,2,3 \ldots
$$


The eigenvalues of the Hamiltonian of the particle inside the small well are equal to

$$
\tilde{E}_{n}=\frac{\pi^{2} \hbar^{2}}{2 m_{c} a^{2}} n^{2} \text {. }
$$

The scalar product of stationary states (4) and (6) is equal to

$$
\begin{aligned}
& \left\langle E_{m} \mid \tilde{E}_{n}\right\rangle=\int_{0}^{a} \mathrm{~d} x\left\langle E_{m} \mid x\right\rangle\left\langle x \mid \tilde{E}_{n}\right\rangle= \\
& \begin{cases}\frac{-4 \sqrt{2} n}{\pi(m+2 n)(m-2 n)} & \text { for } m=1,3,5 \ldots \\
\frac{\sqrt{2}}{2} & \text { for } m=2 n, \\
0 & \text { for } m=2,4,6 \cdots \neq 2 n .\end{cases}
\end{aligned}
$$

The evolution of the state of the particle after instant enlarging of the well (in time $t=0$ ) can be written as follows:

$$
\begin{gathered}
|\psi(t)\rangle=\hat{U}(t)|\psi(0)\rangle=\sum_{m=1}^{\infty} \hat{U}(t)\left|E_{m}\right\rangle\left\langle E_{m} \mid \psi(0)\right\rangle= \\
\sum_{m=1}^{\infty} \exp \left(-\frac{\mathrm{i} E_{m} t}{\hbar}\right)\left|E_{m}\right\rangle\left\langle E_{m} \mid \Psi(0)\right\rangle,
\end{gathered}
$$

where $\hat{U}(t)$ is the time evolution operator.

If one of the stationary states from the small well $\left(|\psi(0)\rangle=\left|\tilde{E}_{n}\right\rangle\right)$ is chosen as the initial state, we obtain (using Eq. (8)) time evolution of the system in the following form:

$$
\begin{aligned}
& \langle x \mid \psi(t)\rangle=\exp \left(-\frac{\mathrm{i} E_{2 n} t}{\hbar}\right)\left\langle x \mid E_{2 n}\right\rangle\left\langle E_{2 n} \mid \tilde{E}_{n}\right\rangle \\
& +\sum_{j=0}^{\infty} \exp \left(-\frac{\mathrm{i} E_{2 j+1} t}{\hbar}\right)\left\langle x \mid E_{2 j+1}\right\rangle\left\langle E_{2 j+1} \mid \tilde{E}_{n}\right\rangle .
\end{aligned}
$$

Equation (10) describes a periodic evolution with period equal to

$$
T=\frac{16 m_{c} a^{2}}{\pi \hbar} .
$$

It is reversible (unitary) process without increasing the entropy (quantum revival of the wave function).

\section{References}

[1] R. Feynman, Int. J. Theor. Phys. 21, 467 (1982).

[2] P.W. Shor, IEEE Comp. Soc. Pr. 124, 4865020 (1994).

[3] L.K. Grover, Am. J. Phys. 69, 769 (2001).
[4] S. Lloyd, Science 273(5278), 1073 (1996).

[5] T. Schaetz, C.R. Monroe, T. Esslinger, New J. Phys. 15, 085009, (2013).

[6] B.P. Lanyon, C. Hempel, D. Nigg et al., Science 334, 57 (2011).

[7] A.M. Childs, D. Maslov, Y. Nam, N.J. Ross, Y. Su, PNAS 115, 38 (2018).

[8] D. Wecker, M.B. Hastings, N. Wiebe, B.K. Clark, C. Nayak, M. Troyer, Phys. Rev. A 92, 062318, (2015).

[9] C. Kokail, C. Maier, R. van Bijnen, et al., Nature 569, 355 (2019).

[10] D. Wecker, B. Bauer, B.K. Clark, M.B. Hastings, M. Troyer, Phys. Rev. A 90, 022305, (2014).

[11] C. Hempel, C. Maier, J. Romero, et al., Phys. Rev. $X$ 8, 031022 (2018).

[12] S.P. Jordan, K.S.M. Lee, J. Preskill, Science 336 , 1130 (2012)

[13] M. Ostrowski, Bull. Pol. Acad. Sci. Techn. Sci. 63 , 379 (2015).

[14] M. Ostrowski, Open Syst. Informat. Dynam. $\mathbf{2 3}$ 1650020 (2016)

[15] S. Wiesner, arXiv:quant-ph/9603028 (1996).

[16] C. Zalka, Fortschr. Phys. 46, 877 (1998).

[17] G. Strini, Fortschr. Phys. 50, 171 (2002).

[18] G. Benenti, Am. J. Phys. 76, 657 (2008).

[19] J.R. Tucker, T.C. Shen, Quant. Inf. Proc. 3, 105 (2005).

[20] D. Loss, D.P. DiVincenzo, Phys. Rev. A 57, 120 (1998).

[21] J. Łuczak, B.R. Bułka, Acta Phys. Pol. A 133, 748 (2018).

[22] G.P. Collins, Sci. Am., (2006).

[23] J. Preskill, opological quantum computation.

[24] T. Maciażek, Acta Phys. Pol. A 136, 824 (2019).

[25] J. Yepez, B. Boghosian, Comp. Phys. Commun. 146, 280 (2002).

[26] J. Yepez, G. Vahala, L. Vahala, Quant. Inf. Proc. 4, 457 (2006).

[27] G. Berman, A.A. Ezhov, I. Kamenev, J. Yepez, Phys. Rev. A 66, 012310 (2002).

[28] M. Ostrowski, "Loading initial data into the quantum register", SMC 2013.

[29] L. Grover, T. Rudolph, arXiv:quant-ph/0208112 (2002)

[30] D. Ventura, Learning quantum operators, in: Proc. Int. Conf. on Computational Intelligence and Neuroscience, 2000 p. 750 . 